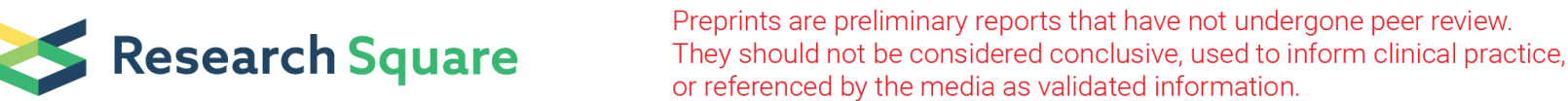

\section{Adding Ursodeoxycholic Acid to the Endoscopic Treatment and Common Bile Duct Stenting for Large and Multiple Biliary Stones: Will it Improve the Outcomes?}

\author{
Ahmad Hormati \\ Qom University of Medical Science \\ Mohammad Reza Ghadir \\ Qom University of Medical Science
}

Seyed Saeed Sarkeshikian

Qom University of Medical Science

Faezeh Alemi ( $\square$ faezeh.alemi@gmail.com )

Qom University of Medical Science

Majid Moghaddam

Qom University of Medical Sciences

\section{Sajjad Ahmadpour}

Qom University of Medical Sciences and Health Services

Abolfazl Mohammadbeigi

Qom University of Medical Sciences

Gholam Reza Sivandzadeh

Shiraz University of Medical Sciences

Research article

Keywords: Cholelithiasis, Gallstones, Common Bile Duct, Ursodiol, Cholangiopancreatography, Endoscopic Retrograde, Sphincterotomy, Endoscopic

Posted Date: September 17th, 2020

DOl: https://doi.org/10.21203/rs.3.rs-67766/v1

License: (1) This work is licensed under a Creative Commons Attribution 4.0 International License.

Read Full License 
Version of Record: A version of this preprint was published at BMC Gastroenterology on November 10th, 2020. See the published version at https://doi.org/10.1186/s12876-020-01523-5. 


\section{Abstract}

Background and Objectives: The role of common bile duct (CBD) stenting in the establishment of bile stream in the elderly patients and the ones who are not good candidates for surgery due to not responding to treatments was well documented in previous studies. The current study aimed at investigating the effect of adding Ursodeoxycholic acid (UDCA) to CBD stenting alone in order to reduce the size or number of large and multiple CBD stones.

Materials and Methods: Clinical outcomes including success rates in CBD stones clearance, incidence of pancreatitis, perforation, bleeding, as well as, decrease in size and number of stones and liver enzymes after a two-month period were assessed in the UDCA + CBD stenting group.

Results: A total of 64 patients referring to Shahid Beheshti University Hospital in Qom, Iran with multiple or large CBD stones (above three or larger than $15 \mathrm{~mm}$ ) received standard endoscopic therapies and UCDA + CBD stenting (group A) and controls only received standard endoscopic therapies + CBD stenting (group B). The mean reduction in the size of stones in group A was significantly higher than that of group $B(3.22 \pm 1.31$ vs $4.09 \pm 1.87 \mathrm{~mm})(p=0.034)$. There was no difference in the incidence rate of complications including pancreatitis, cholangitis, bleeding, and perforation between the two groups $(P$ $>0.05$ ).

Conclusion: Adding UDCA to CBD stenting, due to decrease in the stone size and subsequently facilitation of the stones outlet, can be considered as the first-line treatment for patients with large and multiple CBD stones. Also, in the cases with large or multi stones may be effective in reducing size and subsequently stone retrieval.

\section{Trial registration number:}

The study protocol was approved by the Ethics Committee of Qom University of Medical Sciences (ethical code: IR.MUQ.REC.1397.075); the study was also registered in the Iranian Registry of Clinical Trials (No. IRCT20161205031252N8). This study adheres to CONSORT guidelines.

\section{Background}

Common bile duct (CBD) stone is found in approximately $7 \%-12 \%$ of patients undergoing cholecystectomy for symptomatic gallstones, and is a common indication for endoscopic retrograde choangiopancreatography (ERCP). The CBD stones vary in size, from 1-2 $\mathrm{mm}$ to above $3 \mathrm{~cm}$ in diameter. ERCP with endoscopic sphincterotomy and stone retrieval with basket and balloon is the therapeutic options commonly used to treat CBD stones. It is estimated that roughly $85 \%-95 \%$ of all CBD stones can be effectively treated with these common methods (1).

CBD stones with a maximum diameter of $1.5 \mathrm{~cm}$ can be removed with the endoscopic sphincterotomy technique. By increasing the size of stone, the success rate of these methods and the risk of 
complications such as perforation, cholangitis, and pancreatitis are increased; therefore, the utilization of methods with maximum success rates and minimum complications seems essential. Stones $\geq 15 \mathrm{~mm}$ should be broken up before extraction (2-4).

To treat stones called complicated stones, several methods including various lithotripsy techniques (electrohydraulic lithotripsy (EHL) and laser lithotripsy (LL), endoscopic papillary balloon dilatation (EPBD), sphincterotomy, and CBD stenting are applied. According to the results of different studies, EHL is associated with increased risk of duct rupture and EPBD with increased risk of pancreatitis. In older patients with concomitant critical illnesses that surgical procedures or other endoscopic measures may threaten their lives, the use of less invasive methods with minimal complications seems logic $(5,6)$.

Hydrophilic bile acids, such as ursodeoxycholic acid (UDCA), are used to treat CBD stones, especially cholesterol containing stones. Although the treatment of choice for cholelithiasis is cholecystectomy, patients with small cholesterol stones, without severe symptoms, and proper function of the gallbladder receive UDCA therapy, if they are not good candidates for surgery. Studies indicate that up to $60 \%$ of patients have a chance to completely recover from CBD stones with pharmacotherapies if they are good candidates for medical treatment $(7,8)$.

Regarding the high risk of complications in patients with large and multiple CBD stones, it is reasonable to adopt therapeutic methods with maximum efficacy in order to reduce the size of the stones. Several studies indicated the efficacy of CBD stenting following the endoscopic treatment to reduce the size of large CBD stones. The current study aimed at investigating the effect of adding UDCA to CBD stenting alone in order to reduce the size of stones and increase the success rate of endoscopic treatment in patients with large and multiple CBD stones (9).

\section{Methods}

This randomized, controlled, clinical trial study was conducted on patients with multiple $(\geq 3)$ and/or large (> $15 \mathrm{~mm}$ in diameter) CBD stones. The statistical sample was comprised of patients with large and multiple CBD stones that were candidates for endoscopic treatment by ERCP. The inclusion criteria were both $\geq 3 \mathrm{CBD}$ stones and with $\geq 15 \mathrm{~mm}$ in diameter. The exclusion criteria were history of stomach, duodenum, or CBD surgery, having gastrointestinal stenosis, hemorrhagic disorders, high-risk cardiovascular diseases, and unwillingness to participate in the study.

The sample size required for the current study was determined 32 in each group using the sample size formula, based on the type 1 error as 0.05 and the type 2 error as 0.20 .

The eligible patients after signing the informed consent form were randomly assigned to each of the therapeutic group A or B using the permuted block randomization method. Group A underwent endoscopic therapy with ERCP and stent insertion, and the group B, in addition to ERCP and stenting, received UDCA therapy for eight weeks. Treatments were appointed to groups $A$ and $B$ by coin tossing. In the current study, 10-Fr plastic stents, 8-14 cm length, were used for the subjects based on their CBD 
length. UDCA tablets $300 \mathrm{mg}$ (Tehran Pharm Company) were administered to the subjects three times daily per os.

The information of patients in both groups were recorded in a checklist including demographic characteristics such as age, gender, height, and weight, history of underlying diseases, history of pancreatitis, and the size and number of CBD stones (measured before ERCP). The liver transaminases, alkaline phosphatase, and serum bilirubin levels were measured before the procedure and recorded in the patient's checklist. Patients then underwent ERCP. ERCPs were conducted by three expert gastroenterologists. The number and size of the stones were recorded by fluoroscopy during the ERCP, after the injection of contrast material and radiography. The CBD stent was then inserted. Information on complications during the procedure including perforation of the $\mathrm{CBD}$ and bleeding was recorded in the patient's checklist.

Patients in group B were treated with UDCA and both groups underwent ERCP again after eight weeks in order to remove the stent. In the second turn of the ERCP, complications were monitored during the procedure and after 24 hours. The success rate, defined as a reduction in the number and/or size of CBD stones, was evaluated through ERCP and the results were recorded in the checklist.

Data were analyzed with SPSS 18 software using chi-square, independent and paired samples t-test, and analysis of covariance (ANCOVA). Mean and standard deviation were used to express the data. The Pvalue $<0.05$ was considered as the level of significance.

\section{Results}

Out of 64 patients enrolled in the current clinical trial, Thirty two cases in the control group underwent CBD stenting alone (group A) and the other 32 cases in the intervention group underwent CBD stenting + UDCA (group B). There were 16 females in each group and there was no difference in gender distribution between the groups. The mean age of patients in groups A and B was $44.31 \pm 12.55$ and $41.33 \pm 41.3$ years, respectively. The demographic variables including age and BMI were not significantly different between the two groups. The average number of stones in groups $A$ and $B$ was $3.34 \pm 2.06$ and $3.56 \pm$ 2.26 that was similar in the two groups $(P=0.67)$. The findings are shown in Table 1 . The indication for ERCP in all patients was biliary obstruction due to stone and no evidence of malignancy was detected in cases. Mean levels of liver transaminases, bilirubin, and alkaline phosphatase summarized in Table 2 showed no significant differences between the two groups. 
Table 1

Findings and complications in both groups

\begin{tabular}{|c|c|c|c|c|}
\hline \multicolumn{2}{|c|}{ Findings and Complications } & \multirow{2}{*}{$\begin{array}{l}\text { Control } \\
\text { N (\%) }\end{array}$} & \multirow{2}{*}{$\begin{array}{l}\text { Intervention } \\
\mathbf{N}(\%)\end{array}$} & \multirow[t]{2}{*}{ P-value* } \\
\hline & & & & \\
\hline \multirow[t]{4}{*}{ Pancreatitis } & No & $29(90.6)$ & $27(87.1)$ & \multirow[t]{4}{*}{0.880} \\
\hline & Mild & $2(6.2)$ & $3(9.7)$ & \\
\hline & Moderate & $1(3.1)$ & $1(3.1)$ & \\
\hline & Severe & 0 & 0 & \\
\hline \multirow[t]{2}{*}{ Cholangitis } & No & $31(96.9)$ & $31(96.9)$ & \multirow[t]{2}{*}{1} \\
\hline & Yes & $1(3.1)$ & $1(3.1)$ & \\
\hline \multirow[t]{2}{*}{ Perforation } & No & $32(100)$ & $32(100)$ & \multirow[t]{2}{*}{1} \\
\hline & Yes & 0 & 0 & \\
\hline \multirow[t]{2}{*}{ Sphincterotomy } & No & $32(100)$ & $32(100)$ & \multirow[t]{2}{*}{1} \\
\hline & Yes & 0 & 0 & \\
\hline \multirow[t]{2}{*}{ Stone removal } & No & $27(84.4)$ & $30(93.8)$ & \multirow[t]{2}{*}{0.230} \\
\hline & Yes & $5(15.6)$ & $2(6.2)$ & \\
\hline \multirow[t]{2}{*}{ Diverticula } & Yes & $28(87.5)$ & $26(81.2)$ & \multirow[t]{2}{*}{0.491} \\
\hline & No & $4(12.5)$ & $6(18.8)$ & \\
\hline \multirow[t]{2}{*}{ PD stent } & Yes & $2(6.2)$ & $3(9.4)$ & \multirow[t]{2}{*}{0.641} \\
\hline & No & $30(93.8)$ & $29(90.6)$ & \\
\hline \multirow[t]{2}{*}{ PD Cannulation } & Yes & $25(78.1)$ & $30(93.8)$ & \multirow[t]{2}{*}{0.154} \\
\hline & No & $5(15.6)$ & 2. $(6.2)$ & \\
\hline \multirow[t]{2}{*}{ GIB } & Yes & 5 (16.6) & $3(9.4)$ & \multirow[t]{2}{*}{0.450} \\
\hline & No & $27(84.4)$ & $29(90.6)$ & \\
\hline
\end{tabular}


Table 2

comparsion of liver tests and reduction in the size of stone between two groups

\begin{tabular}{|llll|}
\hline Variable & Control & Intervention & P-value $^{*}$ \\
\hline Reduce in the stone size & $3.22 \pm 1.31$ & $4.09 \pm 1.87$ & 0.034 \\
\hline ALT $^{\#}$ & $184.38 \pm 134.44$ & $196.34 \pm 114.5$ & 0.754 \\
\hline AST $^{+}$ & $141.31 \pm 99.72$ & $153.97 \pm 114.5$ & 0.639 \\
\hline Total Bilirubin & $5.78 \pm 1.64$ & $5.47 \pm 1.98$ & 0.495 \\
\hline Direct Bilirubin & $4.13 \pm 1.18$ & $4.06 \pm 1.64$ & 0.862 \\
\hline Amylase & $120.8 \pm 172.29$ & $127.19 \pm 167.3$ & 0.881 \\
\hline * Based on Independent T-test & & & \\
\hline \# Alanine aminotransferase & & & \\
\hline + Aspartate aminotransferase & & & \\
\hline
\end{tabular}

CBD cannulation was successfully performed in $86 \%$ of the patients and the success rate in the two groups had no difference $(P=0.154)$. The rate of complications during and after ERCP, including pancreatitis, cholangitis, and bleeding, summarized in Table 1, showed no significant differences between the two groups. ERCP-related perforation was not observed in any of the groups.

The average size of stones after eight weeks in groups $A$ and $B$ was $3.22 \pm 1.31$ and $4.09 \pm 1.87 \mathrm{~mm}$, respectively that was significantly higher in group $B(P=0.034)$. The success rate of $C B D$ stones clearance was $27(84.4 \%)$ in group A and $30(93.8 \%)$ in group B. There was no significant difference between two groups in terms of stone clearance rate $(P=0.230)$.

\section{Discussion}

CBD stones vary in size, and most of them are treated with standard procedures. However, in less than $50 \%$ of cases, endoscopic sphincterotomy is difficult because of abnormal anatomy of the duct, abnormal stone location, large size, and high number of stones are effective in this condition $(1,2,10-$ 13). Several methods are proposed for the treatment of large stones of which endoscopic CBD stenting is considered as an effective alternative $(1,5)$. Reduction in the size and number of CBD stones is reported after two months of inserting the stent with a high success rate $(1,5,9)$.

A number of studies showed the efficacy of pharmacotherapy in the treatment of CBD stones with hydrophilic bile acids, especially UDCA. It is also used in the treatment of chronic cholestatic diseases, such as primary biliary cirrhosis (PBC), as a useful and effective therapeutic option to protect the liver and slowdown the progression of liver damage. UDCA inhibits biliary cholesterol secretion, decreases 
intestinal absorption of cholesterol, increases hepatocyte bile secretion, and improves the evacuation of gallbladder of bile and other constituents. It also improves the contraction of gallbladder muscle and reduces inflammation in its wall. However, gallstones are treated medically with UDCA in cases with the mild clinical symptoms, stones smaller than 5-10 mm in diameter, and appropriate function of the gallbladder. The presence of calcium salts in the gallstones, observed as calcification in CT images, reduces the efficacy of UCDA therapy (14-17). However, clinical studies show that if good candidates for medical treatment are selected, up to $60 \%$ are completely cleared of CBD stones after 12-24 months of treatment $(7,8)$.

The current study aimed at examining the efficacy of the combination of two methods of stenting and UDCA therapy in reducing the size of large and number of multiple CBD stones. The results showed that the utilized method could reduce the size of stones by $4 \mathrm{~mm}$ in average that was significantly higher than that of the control group. Also, the number of stones removed from CBD in the intervention group was higher than the control group; however, the difference was statistically insignificant. However, the mean reduction in the number of $\mathrm{CBD}$ stones in the intervention group was higher than that of the control group.

Pancreatitis is one of the most important diseases that predispose ERCP to complications. The overall incidence of pancreatitis was $11 \%$ in the present study, with three cases in the control group and four in the intervention group. There was no significant difference in the incidence of pancreatitis between the two groups. Pancreatitis is identified as the most common post-ERCP complication, and its prevalence generally varies from $3-5 \%$ in studies, but also can vary from $1-16 \%$ depending on selected patients (14-16). In the present study, considering the patients conditions with large and multiple stones, procedures were generally more risky and the high rate of pancreatitis was expected in comparison with the total reported rate.

Cholangitis was observed in two patients (3.13\%), of which one was in the control group and the other in the intervention group. The incidence rate of cholangitis was also different in studies; it was $3.8 \%$ in the study by Hong (17) that was similar to that of the current study, but in the study by Horiuchi (18) it was $13 \%$; the different results can be attributed to patients' differences in various studies.

In the recent study, the success rate in complete clearance of CBD stones was $93.8 \%$ in the intervention group and $84.4 \%$ in the control group. The results was consistent with those of previous studies, including Horiuchi (93\%) (18), Hong (94\%) (17), Hui (94.7\%) (19), Fan (95.6\%) (4) and Ye (94.1\%) (6).

\section{Conclusion}

Generally, it can be concluded that prescribing UDCA along with CBD stenting leads to a further reduction in the size of the stones without increasing complications. However, according to the findings, there was no significant difference in CBD stones clearance rate. It should be noted that as a rule, UDCA is used in long-term treatments (at least 12-24 months), and such circumstances lead to a significant reduction in the size of the stones, especially cholesterol stones. The duration of pharmacotherapy in the current 
study was much shorter than its routine duration (eight weeks vs 12 months). There was also no screening for stones in terms of the type and presence of calcification, especially peripheral calcifications. Given to the fact that UDCA is less effective in cases of calcified stones and patients characteristics is very important in response to UDCA therapy, subsequent clinical trials with larger populations and a more precise selection of patients can be useful in obtaining more conclusive results about the efficacy of the utilized method in the clearance of difficult CBD stones.

\section{Declarations}

\section{Ethics approval:}

The study protocol was approved by the Ethics Committee of Qom University of Medical Sciences (ethical code: IR.MUQ.REC.1397.075).

\section{Consent for publication:}

All the authors have consented for publication.

\section{Availability of data and material:}

All data and materials are available.

\section{Competing interests:}

The authors declare that they have no competing interests.

\section{Funding:}

This work was not supported financially by any institution.

\section{Authors' contributions:}

[Ahmad Hormati], [Mohammad Reza Ghadir] and [Seyed Saeed Sarkeshikian], writing and original draft preparation. [Faezeh Alemi], correspondence(s). [Majid Moghaddam], [Abolfazl Mohammadbeigi] and [Gholam Reza Sivandzadeh] designing, investigation and writing. [Sajjad Ahmadpour], editing the language of the manuscript.

\section{Acknowledgements:}


The authors thank A Jabari, from the Qom University of Medical Sciences, Qom, Iran, for the editing assistance.

\section{References}

1. Freitas ML, Bell RL, Duffy AJ. Choledocholithiasis: evolving standards for diagnosis and management. World journal of gastroenterology. 2006;12(20):3162-7.

2. Disario JA, Freeman ML, Bjorkman DJ, Macmathuna P, Petersen BT, Jaffe PE, et al. Endoscopic balloon dilation compared with sphincterotomy for extraction of bile duct stones. Gastroenterology. 2004;127(5):1291-9.

3. Aslan F, Arabul M, Celik M, Alper E, Unsal B. The effect of biliary stenting on difficult common bile duct stones. Przeglad gastroenterologiczny. 2014;9(2):109-15.

4. Fan Z, Hawes R, Lawrence C, Zhang X, Zhang X, Lv W. Analysis of plastic stents in the treatment of large common bile duct stones in 45 patients. Digestive endoscopy: official journal of the Japan Gastroenterological Endoscopy Society. 2011;23(1):86-90.

5. Yang J, Peng JY, Chen W. Endoscopic biliary stenting for irretrievable common bile duct stones: Indications, advantages, disadvantages, and follow-up results. The surgeon: journal of the Royal Colleges of Surgeons of Edinburgh Ireland. 2012;10(4):211-7.

6. Ye X, Huai J, Sun X. Effectiveness and safety of biliary stenting in the management of difficult common bile duct stones in elderly patients. The Turkish journal of gastroenterology: the official journal of Turkish Society of Gastroenterology. 2016;27(1):30-6.

7. Tangedahl T, Carey WD, Ferguson DR, Forsythe S, Williams M, Paradis K, et al. Drug and treatment efficacy of chenodeoxycholic acid in 97 patients with cholelithiasis and increased surgical risk. Digestive diseases sciences. 1983;28(6):545-51.

8. Maton PN, Iser JH, Reuben A, Saxton HM, Murphy GM, Dowling RH. Outcome of chenodeoxycholic acid (CDCA) treatment in 125 patients with radiolucent gallstones. Factors influencing efficacy, withdrawal, symptoms and side effects and post-dissolution recurrence. Medicine. 1982;61(2):8697.

9. Hormati A, Ghadir M, Sarkeshikian SS, Pezeshki Modarres M, Rafiei M, Alemi F. Efficacy of Common Bile Duct Stenting for Large Stones. 2017. 2017;22(3):5.

10. Samardzic J, Latic F, Kraljik D, Pitlovic V, Mrkovic H, Miskic D, et al. Treatment of common bile duct stones-is the role of ERCP changed in era of minimally invasive surgery? Med Arh. 2010;64(3):1878.

11. Stromberg C, Nilsson M. Nationwide study of the treatment of common bile duct stones in Sweden between 1965 and 2009. Br J Surg. 2011;98(12):1766-74.

12. Binmoeller KF, Bruckner M, Thonke F, Soehendra N. Treatment of difficult bile duct stones using mechanical, electrohydraulic and extracorporeal shock wave lithotripsy. Endoscopy. 1993;25(3):2016. 
13. Lauri A, Horton RC, Davidson BR, Burroughs AK, Dooley JS. Endoscopic extraction of bile duct stones: management related to stone size. Gut. 1993;34(12):1718-21.

14. Cheon YK, Cho KB, Watkins JL, McHenry L, Fogel EL, Sherman S, et al. Frequency and severity of post-ERCP pancreatitis correlated with extent of pancreatic ductal opacification. Gastrointest Endosc. 2007;65(3):385-93.

15. Cotton PB, Garrow DA, Gallagher J, Romagnuolo J. Risk factors for complications after ERCP: a multivariate analysis of 11,497 procedures over 12 years. Gastrointest Endosc. 2009;70(1):80-8.

16. Barthet M, Lesavre N, Desjeux A, Gasmi M, Berthezene P, Berdah S, et al. Complications of endoscopic sphincterotomy: results from a single tertiary referral center. Endoscopy. 2002;34(12):991-7.

17. Hong WD, Zhu QH, Huang QK. Endoscopic sphincterotomy plus endoprostheses in the treatment of large or multiple common bile duct stones. Digestive endoscopy: official journal of the Japan Gastroenterological Endoscopy Society. 2011;23(3):240-3.

18. Horiuchi A, Nakayama Y, Kajiyama M, Kato N, Kamijima T, Graham DY, et al. Biliary stenting in the management of large or multiple common bile duct stones. Gastrointest Endosc. 2010;71(7):12003.e2.

19. Hui CK, Lai KC, Ng M, Wong WM, Yuen MF, Lam SK, et al. Retained common bile duct stones: a comparison between biliary stenting and complete clearance of stones by electrohydraulic lithotripsy. Aliment Pharmacol Ther. 2003;17(2):289-96.

\section{Supplementary Files}

This is a list of supplementary files associated with this preprint. Click to download.

- CONSORTExtensionforwithinpersontrialschecklist.doc 